\title{
Localized surface plasmon fiber device coated with carbon nanotubes for the specific detection of $\mathrm{CO}_{2}$
}

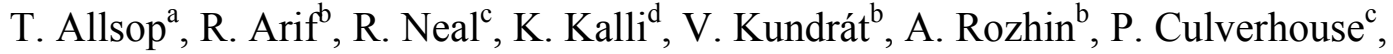 \\ D. J. Webb ${ }^{\mathrm{a}}$ \\ ${ }^{a}$ Aston Institute of Photonic Technologies, School of Engineering and Applied Science, Aston \\ University, Aston Triangle, Birmingham, B47ET, UK; ${ }^{\mathrm{b}}$ Nanoscience Research Group, School of \\ Engineering and Applied Science, Aston University, Aston Triangle, Birmingham, B47ET, UK; \\ ${ }^{\mathrm{c}}$ School of Maths and Computing, Faculty of Science and Technology, University of Plymouth, \\ Plymouth, PL4 8AA, U.K; ${ }^{\mathrm{d} D e p a r t m e n t ~ o f ~ E l e c t r i c a l ~ E n g i n e e r i n g ~ a n d ~ I n f o r m a t i o n ~ T e c h n o l o g y, ~}$ \\ Cyprus University of Technology, Limassol 3036, Cyprus
}

\begin{abstract}
We explored the potential of a carbon nanotube (CNT) coating working in conjunction with a recently developed localized surface plasmon (LSP) device (based upon a nanostructured thin film consisting of of nano-wires of platinum) with ultra-high sensitivity to changes in the surrounding index. The uncoated LSP sensor's transmission resonances exhibited a refractive index sensitivity of $\Delta \lambda / \Delta \mathrm{n} \sim-6200 \mathrm{~nm} / \mathrm{RIU}$ and $\Delta \mathrm{I} / \Delta \mathrm{n} \sim 5900 \mathrm{~dB} / \mathrm{RIU}$, which is the highest reported spectral sensitivity of a fiber optic sensor to bulk index changes within the gas regime. The complete device provides the first demonstration of the chemically specific gas sensing capabilities of CNTs utilizing their optical characteristics. This is proven by investigating the spectral response of the sensor before and after the adhesion of CNTs to alkane gases along with carbon dioxide. The device shows a distinctive spectral response in the presence of gaseous $\mathrm{CO}_{2}$ over and above what is expected from general changes in the bulk refractive index. This fiber device yielded a limit of detection of $150 \mathrm{ppm}$ for $\mathrm{CO}_{2}$ at a pressure of one atmosphere. Additionally the adhered CNTs actually reduce sensitivity of the device to changes in bulk refractive index of the surrounding medium. The polarization properties of the LSP sensor resonances are also investigated and it is shown that there is a reduction in the overall azimuthal polarization after the CNTs are applied. These optical devices offer a way of exploiting optically the chemical selectivity of carbon nanotubes, thus providing the potential for real-world applications in gas sensing in many inflammable and explosive environments.
\end{abstract}

Keywords: Gas-sensing, localized surface plasmons, carbon nanotubes, fiber optic sensors, nanostructured thin film

\section{INTRODUCTION}

The identities of the persons responsible for the discovery of carbon nanotubes ${ }^{1}$ (CNTs) is the subject of much debate but these carbon structures did start to have importance in the 1990s. A lot of research activity has grown around CNTs due to due to their interesting and unusual physical properties such as electrical, optical, mechanical and thermal characteristics $^{2,3}$. Researchers have investigated the versatility of CNTs to be specifically chemically functionalized; this has produced a wide range of specific chemicals that the CNTs can response to or detect ${ }^{4-6}$. Furthermore CNTs are thought to be an ideal candidate as the transducer for gas sensing and have been used to detect very low concentrations of gases ${ }^{7-9}$. The sensing transducers' capabilities have usually been investigated by monitoring the changes in electrical properties in the presence of a specific gas or chemical ${ }^{7-9}$, however some of the apparatus used in this approach is bulky and cumbersome to use and there are questions of operational resilience in real-world applications.

An alternative way is the monitoring of the optical properties CNTs in the presence of a chemical. For this approach to be successful two criteria must be satisfied. Firstly, there is a need for an optical platform that is easy to manage and maintain, such as optical fiber. Secondly, there should be a sensing mechanism that can detect these optical characteristics. To date this has been problematic because conventional types of fiber sensing mechanisms do not have the sensitivity to register the changes in the CNTs.

Recently our team has developed a generic, ultra-high sensitivity, fast-responding environmental optical fiber sensor that consists on a nano-structured thin film coating resembling an array of nano-wires ${ }^{10}$. This array generates a plane of localized surface plasmons (LSPs), which have already demonstrated the able to detect sub nano-molar concentrations of

Optical Sensing, Imaging, and Photon Counting: Nanostructured Devices and Applications,

edited by Manijeh Razeghi, Dorota S. Temple, Gail J. Brown, Proc. of SPIE Vol. 9555

95550S · @ 2015 SPIE · CCC code: 0277-786X/15/\$18 · doi: 10.1117/12.2187557

Proc. of SPIE Vol. 9555 95550S-1 
a chemical in a solution ${ }^{11}$. Surface plasmon resonance is an important optical phenomenon that involves a resonant transfer of incident propagating light to a surface plasmon mode. Surface plasmons take the form of collective electron oscillations at the interface between a dielectric and metal ${ }^{12}$. Furthermore these devices have been shown to the have the ability to work in both the gaseous and aqueous index regime ${ }^{13}$. The uncoated LSP sensor's transmission resonances exhibited a refractive index sensitivity of $\Delta \lambda / \Delta \mathrm{n} \sim-6200 \mathrm{~nm} / \mathrm{RIU}$ and $\Delta \mathrm{I} / \Delta \mathrm{n} \sim 5900 \mathrm{~dB} / \mathrm{RIU}$, which is the highest reported spectral sensitivity of a fiber optic sensor to bulk index changes within the gas regime. The mechanism behind this ultrahigh sensitivity is still being investigated.

We report for the first time a fiber optic sensor device used to monitor changing optical properties of CNTs as a means to measure the concentration of a specific gas; in this case carbon dioxide. The device yielded a limit of detection of $150 \mathrm{ppm}$ for $\mathrm{CO}_{2}$ at a pressure of one atmosphere. Whilst the overall performance of the sensor is similar to others, the results demonstrate that the CNTs working in conjunction these LSP devices offer a potentially powerful new specific gas sensing approach.

\section{LOCALISED SURFACE PLASMON SENSOR FABRICATION}

The LSP fiber devices were fabricated in three stages ${ }^{13}$. Firstly, a standard SMF fiber was mechanically lapped down to $10 \mathrm{~mm}$ from the core-cladding interface. Secondly, using RF sputtering, a series of coatings was deposited upon the flat of the lapped fiber. These coatings consisted of various layers of germanium $(48 \mathrm{~nm})$, silicon dioxide $(48 \mathrm{~nm})$ and platinum (36nm). Thirdly, the coated fiber was exposed to a UV light interference pattern produced by a uniform phase mask with period $1.018 \mu \mathrm{m}$ through laser beam scanning and multi-exposure, see figure 1a. Using an Atomic Force Microscope (AFM) it was found that coatings consisted of an array of metal nano-wires (typically $36-\mathrm{nm}$ in radius and $20 \mu \mathrm{m}$ in length), supported by a silicon dioxide thin film on a thin substrate of germanium, the nano-wires being perpendicular to the longitudinal axis of the D-shaped fiber, see figure $1 \mathrm{~b}$. This produced a surface relief structure which has dominant spatial periods close $\sim 0.5 \mu \mathrm{m}$ and $\sim 1 \mu \mathrm{m}$, see figure $2 \mathrm{a}$. The mechanism that creates this corrugation is still being investigated; there is some evidence suggesting that the UV irradiance generates the surface topology by the creation of germanium oxides (by a photo-bleaching process) that produces a periodic stress field governing the growth of these structures ${ }^{10}$. The consequence of the UV $(244 \mathrm{~nm})$ exposure on a planar germanium sample is shown in Figure2b.
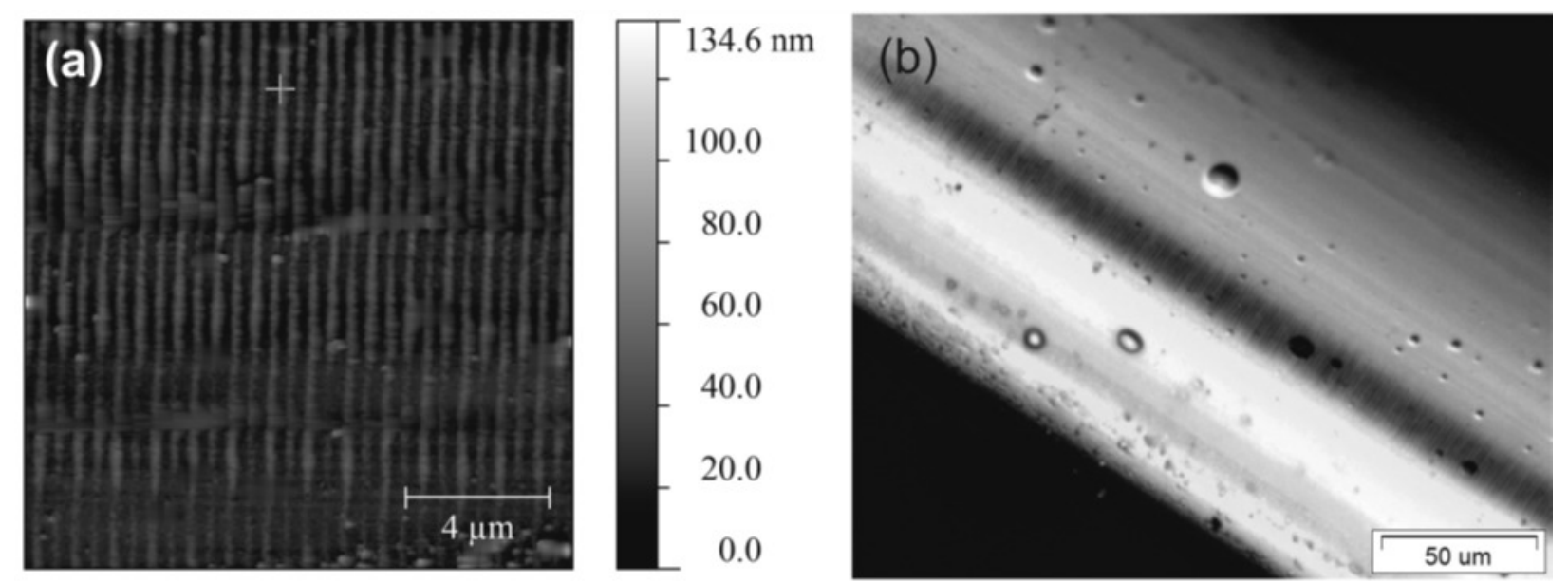

Figure 1 Images and topological data of the post UV-laser processed platinum fiber device: (a) an AFM image; (b) a visible microscope image. 

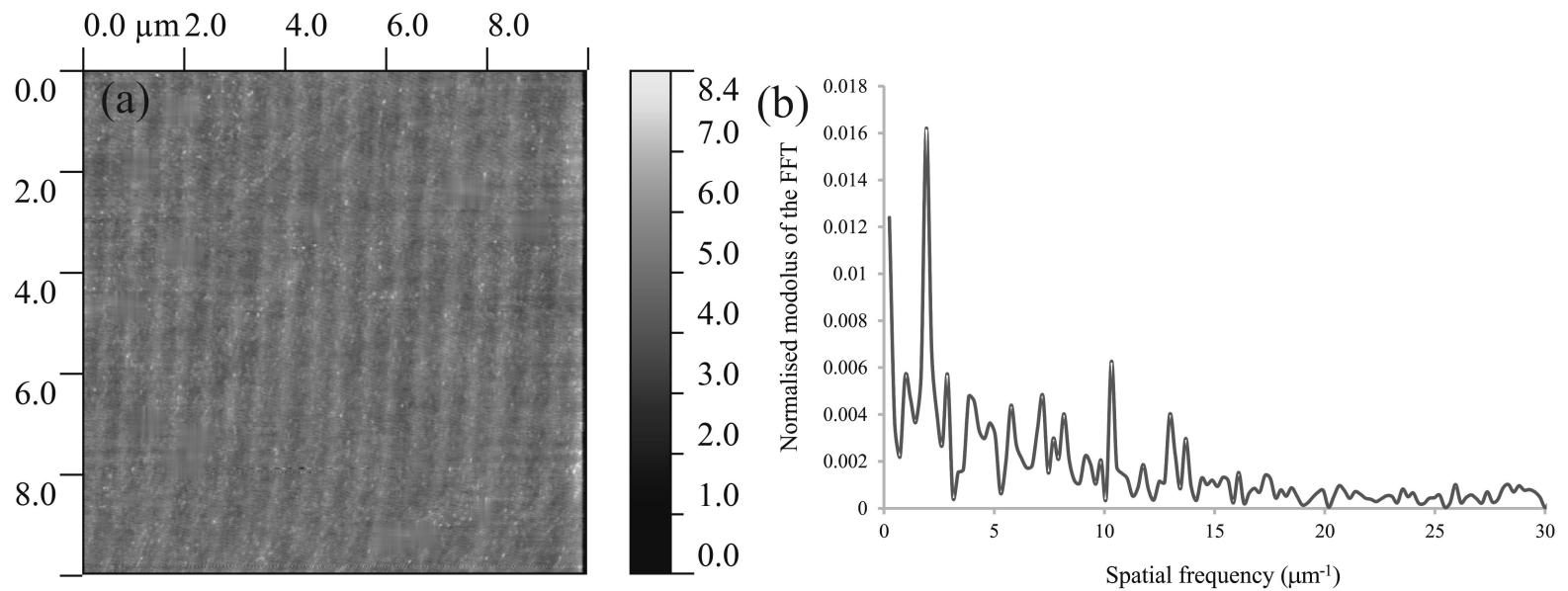

Figure 2 (a) view of the AFM data showing an example of the effect of a UV coherent laser light (argon ion laser at $244 \mathrm{~nm}, 110 \mathrm{~mW}$ of power, scan velocity of $0.05 \mathrm{mms}^{-1}$, and an optical phase mask with a period of $1.018 \mu \mathrm{m}$ ) on the surface topology of a thin coating consisting of germanium (48nm) and (b) a fast Fourier transform (FFT) of the typical AFM line profile of the post-UV processed coating.

\section{SPECTRAL CHARACTERISATION OF THE LSP SENSOR}

Firstly, these devices were characterized by measuring changes in their response as a function of the azimuthal polarization properties of the illuminating light and the changes in the surrounding medium's refractive index Fibers were placed in a V-groove and immersed in certified refractive index (CRI) liquids that have a quoted accuracy of \pm 0.0002 , in the aqueous index regime. Figure 3 shows how the spectral sensitivity changes with the UV processing of the multi-layered coating. Due to the spectral broadness of these transmission features, the central wavelength is calculated by the first moment of the power spectrum; the centroid by geometric decomposition ${ }^{14}$. The centroid is given by:

$$
\lambda_{\text {cent }}=\frac{\int_{\lambda_{s}}^{\lambda_{f}} \lambda \cdot I(\lambda) d \lambda}{\int_{\lambda_{s}}^{\lambda_{f}} I(\lambda) d \lambda},
$$

where $\lambda_{\text {cent }}$ is the centroid wavelength over a range of $\lambda_{\mathrm{s}}$ to $\lambda_{\mathrm{f}}$ and $I$ are the associated amplitude/intensities measured in $\mathrm{dBs}$ over the chosen part of the spectrum at $90 \%$ of the maximum coupling strength, where the surface plasmon resonance exists. The associated centroid strength value is taken as the mean value over the interval range of interest

There are several surface plasmon resonances observed at different spectral locations depending upon the azimuthal polarization condition of the illuminating light, see figure 3a. For example, rotating the azimuth of polarization from the optimum reduced the strength of the resonance $\sim 2.6 \mathrm{~dB} /$ degree, see figure $3 \mathrm{~b}$. It can be seen from figure 4 that the spectral sensitivity to changes in the surrounding refractive index has dramatically increased. Before UV processing the highest index sensitivity measured was $\Delta \lambda / \Delta \mathrm{n} \sim 2100 \mathrm{~nm} / \mathrm{RIU}$, while after the UV the maximum observed sensitivity was $\Delta \lambda / \Delta \mathrm{n} \sim 10700 \mathrm{~nm} / \mathrm{RIU}$ in the aqueous index regime (1.36 to 1.39), which is approaching an order of magnitude increase in sensitivity. 

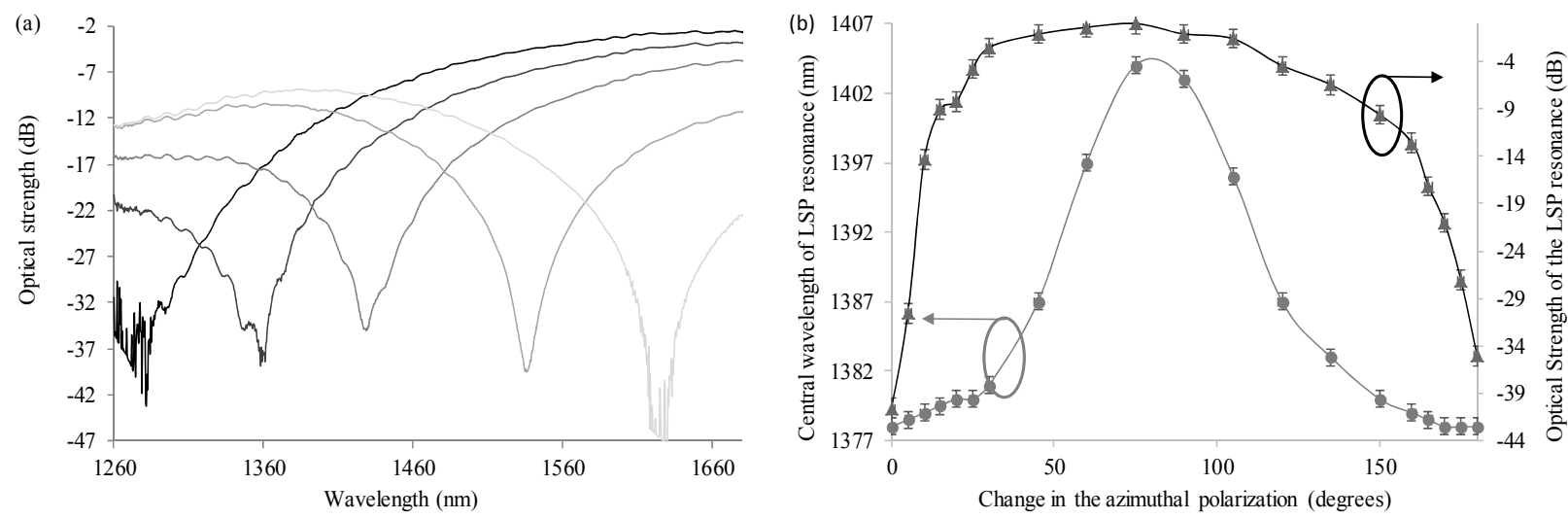

Figure 3 (a) the variation in the plasmon's resonance as a function of the polarization state of the illuminating light. (b) the typical azimuthal polarization dependence of a localized surface plasmon fiber device in air before the coating of CNTs
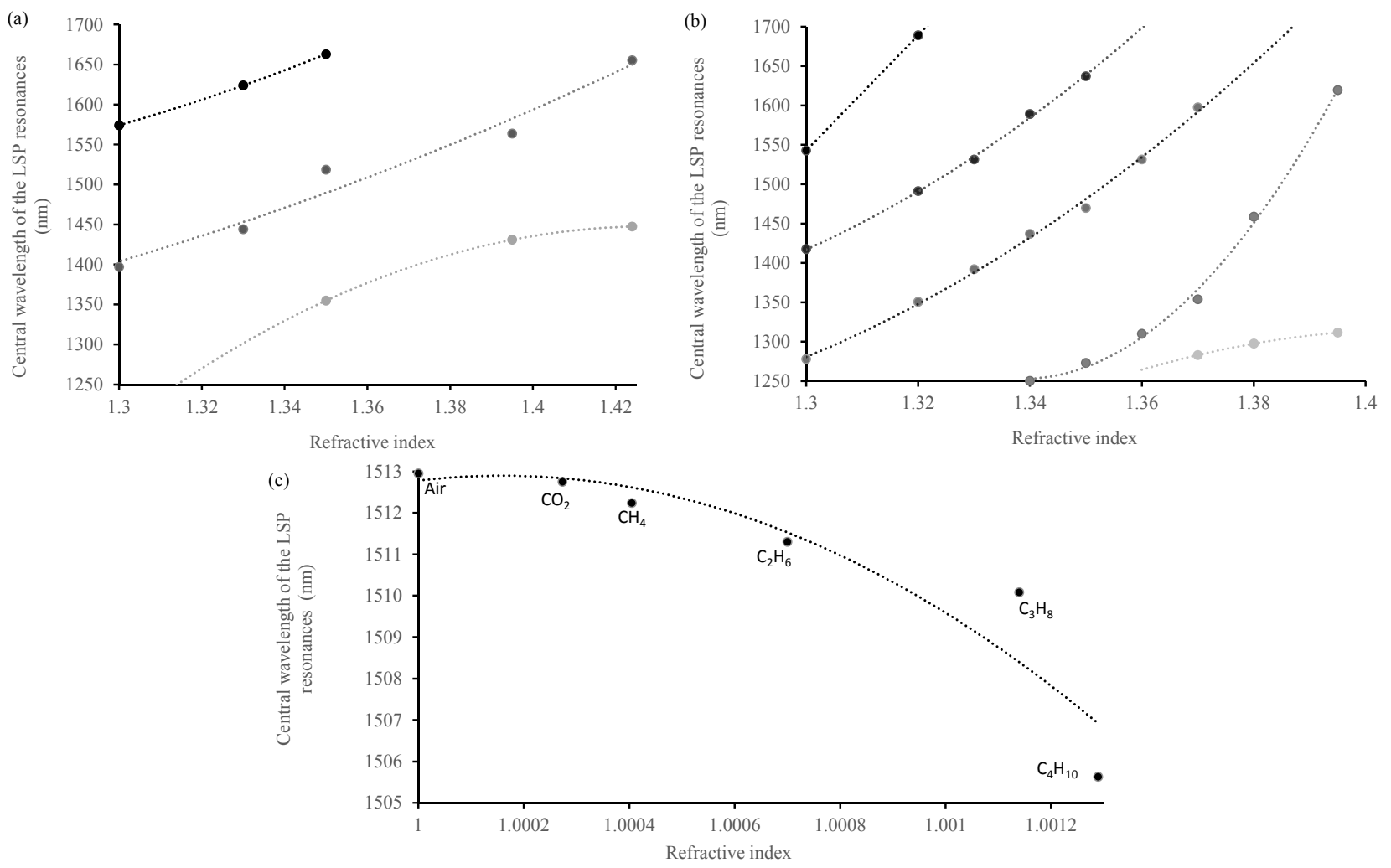

Figure 4 The spectral sensitivities with respect to refractive index in the aqueous regime (a) before UV processing (conventional surface plasmons generated from the multi-layered coating on the optical fiber) (b) after UV processing - the generation of localized surface plasmons. (c) The spectral sensitivity of the resonance at $1510 \mathrm{~nm}$ as a function of low refractive indices, after UV processing.

The sensitivity of the devices at low refractive indices was studied with the alkane gases (methane, ethane, propane and butane). This approach was also used after CNTs were adhered to the LSP sensor. Figure 4c shows the spectral sensitivity of the LSP sensor to the alkane gases and carbon dioxide and reveals a refractive index sensitivity of $\Delta \lambda / \Delta \mathrm{n} \sim$ $-6200 \mathrm{~nm} / \mathrm{RIU}$ and $\Delta \mathrm{I} / \Delta \mathrm{n} \sim 5900 \mathrm{~dB} / \mathrm{RIU}$ in the gaseous index regime for the $1510 \mathrm{~nm}$ resonance. The authors believe that 
this is the highest reported spectral sensitivity of a fiber optical sensor to index changes operating within the gas regime. Following this initial characterization of the LSP, the sensors where coated with CNTs.

\section{SPECTRAL CHARACTERISATION OF THE LSP SENSOR WITH A CNT COATING}

The CNTs coating was attached using wet chemistry, the adhesion process, using purified single wall CNTs (CoMoCaT CG 200, SouthWest NanoTechnologies Inc.), is as follows. Firstly, $0.5 \mathrm{mg}$ of CNTs were dispersed in $10 \mathrm{ml}$ of Nmethyl-2-pyrrolidone (NMP) via sonication $(20 \mathrm{kHz}, 200 \mathrm{~V}, 1$ hour, Nanoruptor, Diagenode SA). The use of NMP is conditioned by its efficiency in the direct dispersion of CNTs (hydrophobic material) at concentrations below 0.02 $\mathrm{mg} / \mathrm{mL}^{15}$. Additionally, we added polyvinyl pyrrolidone (PVP) polymer $(1 \mathrm{mg} / \mathrm{ml})$ as a dispersion agent in order to achieve higher concentrations of CNTs within the resulting dispersion ${ }^{16}$. In order to achieve a highly uniform dispersion and remove residual CNT bundles, the CNT-PVP-NMP system was centrifuged for 30 minutes at 10000 RPM with MLS-50 rotor (Optima MaxXP Benchtop Ultracentrifuge, Beckman Coulter). The SPR fiber device was placed subsequently in the micro-capillary tube filled with the CNT dispersion for a few minutes. Finally, the resulting device was dried in air at atmospheric pressure for 24 hours before being placed in the gas line.

After the CNTs were adhered to the surface of the fiber optic sensor, LSP resonances were observed, with central wavelengths of $1540 \mathrm{~nm}$ and $1430 \mathrm{~nm}$ and with optical strengths of $41 \mathrm{~dB}$ and $50 \mathrm{~dB}$, respectively. The sensor was placed within the gas chamber and the sensor was characterized with respect to spectral index sensitivity using the alkane gases, the specific spectral response to carbon dioxide, the limit of detection to $\mathrm{CO}_{2}$, and its polarization dependence.
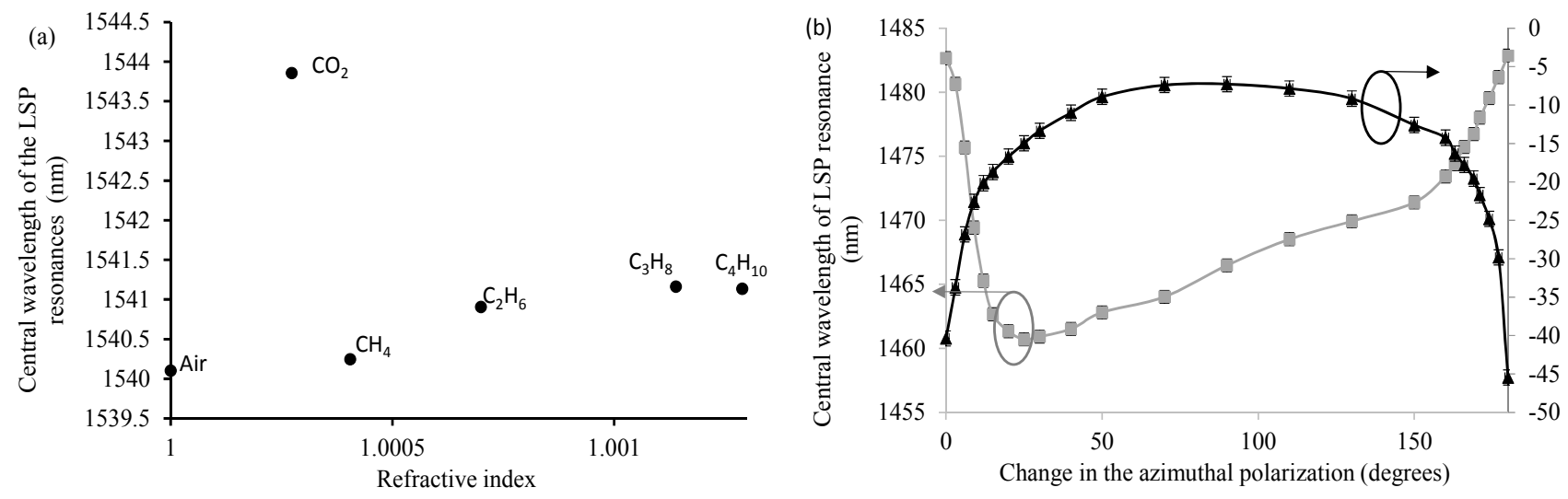

Figure 5 (a) A demonstration of the LSP sensor being functionalized for a specific chemical - in this case carbon dioxide - using a resonance at $1540 \mathrm{~nm}$. (b) A typical azimuthal polarization dependence of a localized surface plasmon resonance of the sensor coated with CNTs.

Inspecting figure 5a, the sensor registers a large wavelength shift $(\Delta \lambda \sim 3.8 \mathrm{~nm})$ in the presence of $\mathrm{CO}_{2}$, compared to the alkane gases when comparing the results obtained prior to the adhesion of the CNTs. The authors also conducted a spectral sensitivity control experiment with a LSP sensor coated with the adhesion agent without CNTs, which yielded no signification wavelength shift above what is expected for the change in bulk refractive index of the submersing material. Therefore this demonstrates a large difference between the response to bulk refractive index and the chemically induced changes in the optical properties, caused by the specific interplay between the $\mathrm{CNTs}$ and $\mathrm{CO}_{2}$. Comparing the polarization dependence before and after the adhesion of the CNTs, there is a reduction from $2.6 \mathrm{~dB} /$ degree before coating and only $1.8 \mathrm{~dB} /$ degree afterwards. It is known that the shape of the supporting particles of the surface plasmons affects the polarization characteristics ${ }^{17}$. The polarization dependence behavior that has been observed suggests that the CNTs are supporting the plasmons or at least that the plasmons are interacting with the CNTs. 
To enable a comparison with other researchers' results, the response provides equivalent spectral index sensitivities in excess of $3 \times 10^{4} \mathrm{~nm} / \mathrm{RIU}$ and $4.2 \times 10^{4} \mathrm{~dB} / \mathrm{RIU}$ when the atmosphere was approaching $100 \% \mathrm{CO}_{2}$, which leads to an equivalent index resolution of approximately $10^{-5}$ using the procedure given in reference ${ }^{18}$ for spectral sensitivity to $\mathrm{CO}_{2}$. In a final experiment, the sensor was continuously monitored during an experiment to observe the wavelength shift in a gas with a composition varying from standard atmospheric conditions to a saturated atmosphere of $\mathrm{CO}_{2}$ with an inlet flow rate of 0.5 litres $/ \mathrm{min}$. This was to estimate the limit of detection and response time of this type of sensor and typical results are shown figure 6. The superimposed slow sinusoidal variation in figure 6 is a repeatable systematic error produced from temperature drift in the experimental apparatus. This test was performed several times on three different sensors with the same fabrication conditions and all showed a selective response to $\mathrm{CO}_{2}$ but with differing spectral sensitivities. Wavelength shifts ranged from $0.6 \mathrm{~nm}$ to $4 \mathrm{~nm}$ over the full range of $\mathrm{CO}_{2}$ concentrations resulting in detection limits from 523ppm to $150 \mathrm{ppm}$ at one atmosphere pressure. The detection limit was obtained by determining the wavelength shift as a function of $\mathrm{CO}_{2}$ concentration and using the spectral resolution of the resonance ${ }^{18}$. The differences in the resolution of each sensor can be attributed to small variations in the manual fabrication procedure used and other experimental and environmental parameters, such as the polarization of the illuminating light, the central wavelength of the LSP resonances and fluctuations in temperature.

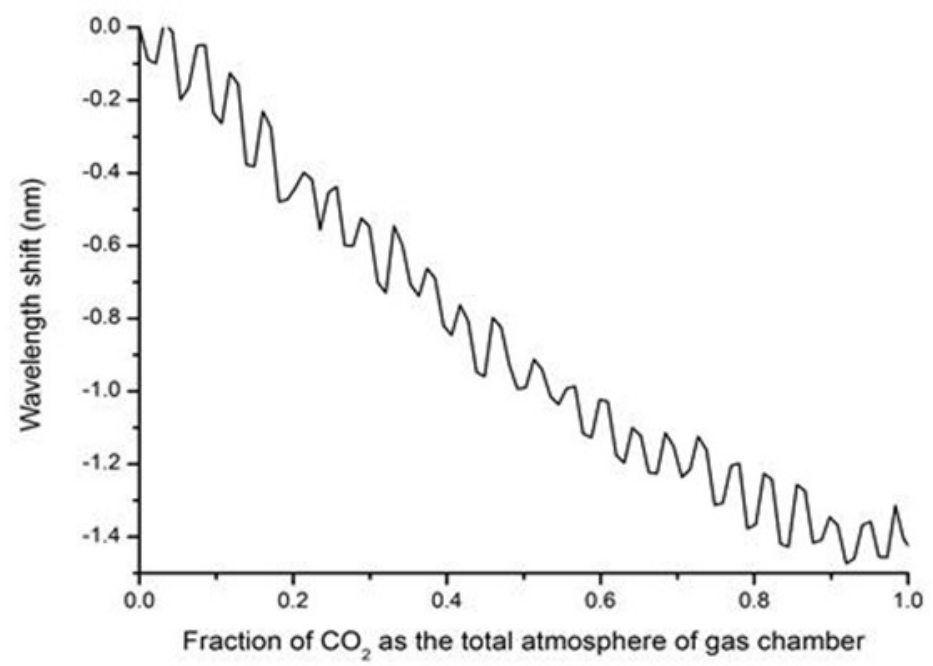

Figure 6 The typical spectral behavior of the fiber sensor with a LSP wavelength resonance at 1390nm as a function of the fraction of $\mathrm{CO} 2$ in the surrounding atmosphere.

\section{CONCLUSION}

We demonstrate an optical fiber plasmonic gas sensor that utilizes a $\mathrm{CO}_{2}$ specific change in the optical properties of CNTs coating the sensing platform. The sensor is able to operate due to the ultra-high sensitivity of the underlying fibre device in the gaseous regime $(\Delta \lambda / \Delta \mathrm{n} \sim-6200 \mathrm{~nm} / \mathrm{RIU})$. This is the first demonstration of species-specific optical fiber gas sensing that utilizes the optical properties of CNTs. The limit of detection of the sensor investigated was 150ppm. This sensor is the first significant step towards the realization of a practical gas sensor based upon the optical properties of CNTs. It is important to stress that functionalization of the CNTs offers the prospect of extending the specific response to other gases ${ }^{4-9}$ 


\section{ACKNOWLEDGMENT}

This work was financially supported by grants EP/J010413 and EP/J010391 for Aston University and University of Plymouth from the UK Engineering and Physical Sciences Research Council. To access the data underlying this publication, please contact researchdata@aston.ac.uk, see http://dx.doi.org/10.17036/Xxxxxxxxxxx-Xxxx-Xxxx-Xxxxxxxxxxxx

\section{REFERENCES}

[1] Lijima, S., "Helical microtubules of graphitic carbon", Nature 354, 56-58 (1991).

[2] M. Ouyang, M., Huang, J.-L., Lieber, C.M., "Fundamental electronic properties and applications of single-walled carbon nanotubes", Acc. Chem. Res. 35, 1018-102, (2002).

[3] Dresselhaus, M. S., Dresselhaus G., Charlier, J. C., Hernandez, E., "Electronic, thermal and mechanical properties of carbon nanotubes", Philosophical Transactions of the Royal Society of London. Series A: Mathematical, Physical and Engineering Sciences 362.1823, 2065-2098, (2004).

[4] Penzaa, M., Rossi, R., Alvisi, M., Cassanoa, G., Signorea, M. A., Serrab, E., Giorgi, R., "Pt- and Pd-nanoclusters functionalized carbon nanotubes networked films for sub-ppm gas sensors", Sensors and Actuators B, 135, 289 297, (2008).

[5] Hirsch, A., "Functionalization of single-walled carbon nanotube", Angewandte Chemie Int. Edition 41.11, 1853$1859,(2002)$.

[6] Hongjie, D., "Carbon nanotubes: synthesis, integration, and properties", Accounts of chemical research, 35.12, 1035-1044 (2002).

[7] Sun, G., et al., "Electrochemical chlorine sensor with multi-walled carbon nanotubes as electrocatalysts", Electrochemistry Communications, 9.9, 2436-2440 (2007).

[8] Bekyarova, E., et al, "Chemically functionalized single-walled carbon nanotubes as ammonia sensors", The Journal of Physical Chemistry B 108.51, 19717-19720, (2004).

[9] Zhang, Y., Zhang, D., Liu, C,. "Novel Chemical Sensor for Cyanides: Boron-Doped Carbon Nanotubes", J. Phys. Chem. B, 110, 4671-4674, (2006).

[10] Allsop, T., et al., "Formation and characterisation of ultra-sensitive surface plasmon resonance sensor based upon a nano-scale corrugated multi-layered coated D-shaped optical fibre”, Jn. Quantum Electronics, 48(3), 394-405, (2012).

[11] Allsop, T., et al., "Aptamer-based surface plasmon fibre sensor for thrombin detection", SPIE Photonics Europe. International Society for Optics and Photonics, 77151C-77151C, (2010).

[12] [Surface Plasmons on smooth and Rough Surfaces and on Gratings], H. Raether, eds. (Academic, New York, 1997).

[13] Allsop, T., et al., "The exploitation of multilayer coatings for infra-red surface plasmon resonance fibre sensors", Appl. Optics 48(1), 276-286 (2009).

[14] [Numerical geometry of non-rigid shapes], A. M. Bronstein, R. Kimmel eds. (Springer Science \& Business Media, 2008).

[15] Giordani, S., et al., "Debundling of single-walled nanotubes by dilution: Observation of large populations of individual nanotubes in amide solvent dispersions" J Phys Chem B. 110, 15708 (2006)

[16] Hasan, T., et al., "Stabilization and "debundling" of single-wall carbon nanotube dispersions in N-methyl-2pyrrolidone (NMP) by polyvinylpyrrolidone (PVP)", J. Phys. Chem. C, 111, 12594 (2007)

[17] Miller, M. M., Lazarides, A. A., "Sensitivity of Metal Nanoparticle Surface Plasmon Resonance to the Dielectric Environment," J. Phys. Chem. B. 109, 21556-21565 (2005).

[18] Hu, J., Sun, X., Agarwal, A., Kimerling, L. C., "Design guidelines for optical resonator biochemical sensors", J. Opt. Soc. Am. B 26, 1032-1041 (2009). 